\title{
Monodromy in the spectrum of a rigid symmetric top molecule in an electric field
}

\author{
I. N. Kozin \\ Department of Chemistry, University of Aberdeen, Meston Walk, Aberdeen AB24 3UE, United Kingdom \\ R. M. Roberts \\ Department of Mathematics and Statistics, University of Surrey, Guildford GU2 7XH, United Kingdom
}

(Received 6 March 2002; accepted 19 March 2003)

\begin{abstract}
We show that for rigid symmetric top molecules in electric fields the phenomenon of monodromy arises naturally as a "defect" in the lattice of quantum states in the energy-momentum diagram. This makes it impossible to use either the total angular momentum or a pendular quantum number to label the states globally. The monodromy is created or destroyed by classical Hamiltonian Hopf bifurcations from relative equilibria. These phenomena are robust and should be observable in quasi-symmetric top molecules with field strengths $\mathcal{E}$ satisfying $\mu \mathcal{E} / b>4.5$, where $\mu$ is the dipole moment and $b$ the rotational constant perpendicular to the symmetry axis of the molecule. (C) 2003
\end{abstract} American Institute of Physics. [DOI: 10.1063/1.1573633]

\section{INTRODUCTION}

A recent major advance in molecular dynamics has been the extensive use of electric fields to orient molecules in order to study spatial effects in reactive scattering, spectroscopy, and photodissociation. ${ }^{1-9}$ This method, known as the brute force technique, was initially applied to linear and diatomic molecules and then extended to symmetric and asymmetric polar molecules. A molecule in a moderately strong electric field exhibits two different regimes: pendular motion about oriented states at low energies and almost free rotational motion at high energies. The former quantum states can be labeled by a pendular quantum number, while the latter are labeled by the usual total angular momentum. This system is a quantum realisation of the Lagrange top which is one of the most fundamental classical systems with monodromy. ${ }^{11,10}$ When the energy levels of the quantum states are plotted against a conserved quantity, such as the projection of the total angular momentum onto the direction of the electric field, then it is observed that some series of levels with the same total angular momentum or same pendular number display a characteristic kink. Moreover, if the constant total angular momentum series are smooth, then the constant pendular quantum number series have a kink and vice versa. A similar kink phenomenon occurs for rotating bending states of quasilinear molecules for which it is possible to use either the quantum labelling for bent molecules $(\nu, K)$ or that for linear molecule $(n, l) .{ }^{12}$

It has been shown that for both linear molecules in electric fields and quasilinear molecules this behavior is due to the presence of monodromy. ${ }^{12,13}$ It should be noted that the monodromy is a universal phenomenon but the kink is not. For integrable classical mechanical systems with two degrees of freedom monodromy is an obstruction to the existence of global action angle variables ${ }^{14}$ and is present whenever the energy-momentum map has a singular fibre in the form of a "pinched torus." 15-18 Monodromy has been studied in a variety of model systems, including the "champagne bottle" or
"Mexican hat" potential, ${ }^{19}$ the spherical pendulum, ${ }^{10,20}$ and the Lagrange top. ${ }^{10,11}$ In quantum systems monodromy is revealed by the lack of a uniform smooth set of quantum numbers for labelling quantum states localized near the pinched torus. This has been demonstrated for the spherical pendulum $^{21}$ and the champagne bottle potential. ${ }^{13}$ Monodromy has also been discovered in one of the most basic atomic systems: hydrogen atom in orthogonal electric and magnetic fields. ${ }^{22}$ A general semiclassical theory relating quantum and classical monodromy is given in Refs. 23, 24 and further applications to atomic and molecular systems in Refs. 25-27.

The appearance of monodromy in classical mechanical systems is closely related to the occurrence of Hamiltonian Hopf bifurcations of relative equilibria. ${ }^{28,29}$ In our case, a relative equilibrium of a molecule is a steady motion in which the molecule rotates about a fixed axis without changing its shape. It is therefore an equilibrium state in an appropriate rotating frame. Relative equilibria have been used as "organizing centers" for molecular spectra at high angular momenta in much the same way as equilibria are used for vibrational and low angular momentum spectra. ${ }^{30-34}$ They appear in families parametrized by total angular momentum and typically undergo a number of bifurcations as the angular momentum is increased. Hamiltonian Hopf bifurcations occur when two normal mode frequencies become equal and then complex, resulting in loss of stability. This is not uncommon: any given rotating molecular system is likely to exhibit several such bifurcations as the angular momentum is increased. $^{33,34}$ Of course molecular systems are not integrable. However integrable approximations often provide useful models. For example, the monodromy present in linear molecules in electric fields and quasilinear molecules can be explained using approximations by the spherical pendulum and champagne bottle potential, respectively. ${ }^{12,13}$ In the case of Hamiltonian Hopf bifurcations normal form theory introduces extra approximate symmetries and conserved 
quantities near the bifurcation point and these can be used to construct local integrable approximations. ${ }^{28}$ None of these approximations will be valid globally.

In this paper we study bifurcations of relative equilibria and the quantum monodromy of a rigid symmetric top with an electric dipole in an electric field. This is an integrable approximation used to model the orientation of quasisymmetric top molecules by electric fields. It is mathematically identical to the Lagrange top, which is known to have a Hamiltonian Hopf bifurcation. ${ }^{10,28,35}$ Its conserved quantities include the angular momenta about both the field axis and the symmetry axis of the body. In applications to quasisymmetric top molecules the angular momentum about the symmetry axis will only be conserved approximately. The paper has several aims, including a description of the quantum monodromy of the Lagrange top using its relative equilibria as a "skeleton," a description of the effect of the Hamiltonian Hopf bifurcation on quantum monodromy, some remarks on perturbations away from complete integrability, and, perhaps most importantly, a discussion of the implications of these results for the spectroscopy of quasisymmetric molecules in electric fields.

In Sec. II we describe the stability and bifurcations of relative equilibria of axisymmetric rigid bodies in electric fields, recalling earlier results on the Lagrange top. ${ }^{11,35-38}$ In Sec. III we present plots of the energies of quantum states against the angular momentum about the symmetry axis of the body and show how the structures observed are organized by the relative equilibrium bifurcation diagrams of Sec. II. This is followed by discussions of the effects of perturbations away from symmetry and the critical field strength needed to observe monodromy.

\section{CLASSICAL THEORY}

The Hamiltonian for a rigid top molecule with a dipole of strength $\mu$ parallel to the molecular $z$-axis in a static electric field of strength $\mathcal{E}$ can be written as

$$
H=a J_{z}^{2}+b J_{x}^{2}+c J_{y}^{2}-\mu \mathcal{E} \cos \theta,
$$

where the $J_{\alpha}$ are the projections of the total angular momentum onto the molecule fixed axis system and the angle $\theta$ is the angle between the molecule $z$-axis and the electric field axis. Small letters are used for the rotational constants to avoid confusion with traditional capital letters which normally assume $A>B>C$. We allow for $a, b$, and $c$ any physically possible values but restrict the $z$-axis to be fixed along the dipole. Then a top which is symmetric about its dipole axis satisfies $b=c$. Following convention, the rotational constants have the dimension of energy while the angular momentum is dimensionless. The Hamiltonian of a symmetric top in electric field is commonly expressed in dimensionless form by

$$
H / b=\mathbf{J}^{2}+(\gamma-1) J_{z}^{2}-\omega \cos \theta,
$$

where $\gamma=a / b$ and the new dimensionless field parameter $\omega=\mu \mathcal{E} / b$. The rotational constant $a$ is half the inverse of the principal moment of inertia about the symmetry axis and $b$ is half the inverse of the orthogonal principal moment of inertia. Prolate molecules are given by $\gamma>1$ and oblate molecules by $\gamma<1$.

Let $\mathbf{n}=\left(n_{x}, n_{y}, n_{z}\right)$ be a unit vector in the field direction referred to the molecular axis system. Note that $n_{z}=\cos \theta$. The Hamiltonian (2) is invariant under both rotations about the field axis in the space fixed frame and rotations of the molecule about its symmetry axis. The corresponding conserved quantities are the angular momenta of the molecule about the two axes, $m=\mathbf{J} . \mathbf{n}$ and $k=J_{z}$, respectively. This makes it possible to reduce the original three-dimensional problem to a one-dimensional system. However in this paper we will usually describe classical bifurcations and quantum spectra by fixing $m$ and allowing $k$ to vary. This makes the problem effectively two dimensional. Such a view is natural since for real molecules $k$ will only be approximately conserved.

\section{A. Relative equilibria}

The classical equations of motion for the Hamiltonian (1) can be written as, ${ }^{10,11,39}$

$$
\begin{aligned}
& \dot{\mathbf{J}}=\mathbf{J} \times \frac{\partial H}{\partial \mathbf{J}}+\mathbf{n} \times \frac{\partial H}{\partial \mathbf{n}}, \\
& \dot{\mathbf{n}}=\mathbf{n} \times \frac{\partial H}{\partial \mathbf{J}} .
\end{aligned}
$$

For an axisymmetric rigid body the relative equilibria are the solutions of these equations for which the motion consists of steady rotation about its symmetry axis combined with steady rotation of the symmetry axis about the field axis. Equivalently, the relative equilibria with angular momenta $J_{z}=k$ about the symmetry axis and $\mathbf{J} . \mathbf{n}=m$ about the field axis are the critical points of $H$ constrained to the surface,

$$
J_{z}=k, \quad \mathbf{J} . \mathbf{n}=m, \quad \mathbf{n} . \mathbf{n}=1 .
$$

A calculation shows that there are three types of relative equilibria satisfying, respectively,

$$
\begin{aligned}
& \text { P: } \quad \mathbf{J}=(0,0, m), \quad \mathbf{n}=(0,0,1), \quad k=m ; \\
& \mathrm{AP}: \quad \mathbf{J}=(0,0, m), \quad \mathbf{n}=(0,0,-1), \quad k=-m ; \\
& \mathrm{T}^{k}: \quad \mathbf{J}=\left(J_{x y} \cos \phi, J_{x y} \sin \phi, k\right), \\
& \mathbf{n}=(\sin \theta \cos \phi, \sin \theta \sin \phi, \cos \theta), \\
& \phi-\operatorname{arbitrary,} \quad J_{x y}=(m-k \cos \theta) / \sin \theta, \\
& \omega \sin ^{4} \theta+2(k \cos \theta-m)(m \cos \theta-k)=0 .
\end{aligned}
$$

The $\mathrm{P}$ and AP relative equilibria are those for which the field and molecule symmetry axes are aligned. For the AP relative equilibrium the dipole is opposite to that of the electric field, while for the $\mathrm{P}$ relative equilibrium they are the same. We therefore refer to them as being antiparallel and parallel, respectively.

For $J_{x y} \neq 0$ the field and symmetry axes of the $\mathrm{T}^{k}$ relative equilibria are not aligned and so we refer to them as tilted relative equilibria. The parallel and antiparallel relative equilibria are limits of families of tilted relative equilibria as $J_{x y} \rightarrow 0$. For $k \neq \pm m$ there exists a unique tilted relative equi- 


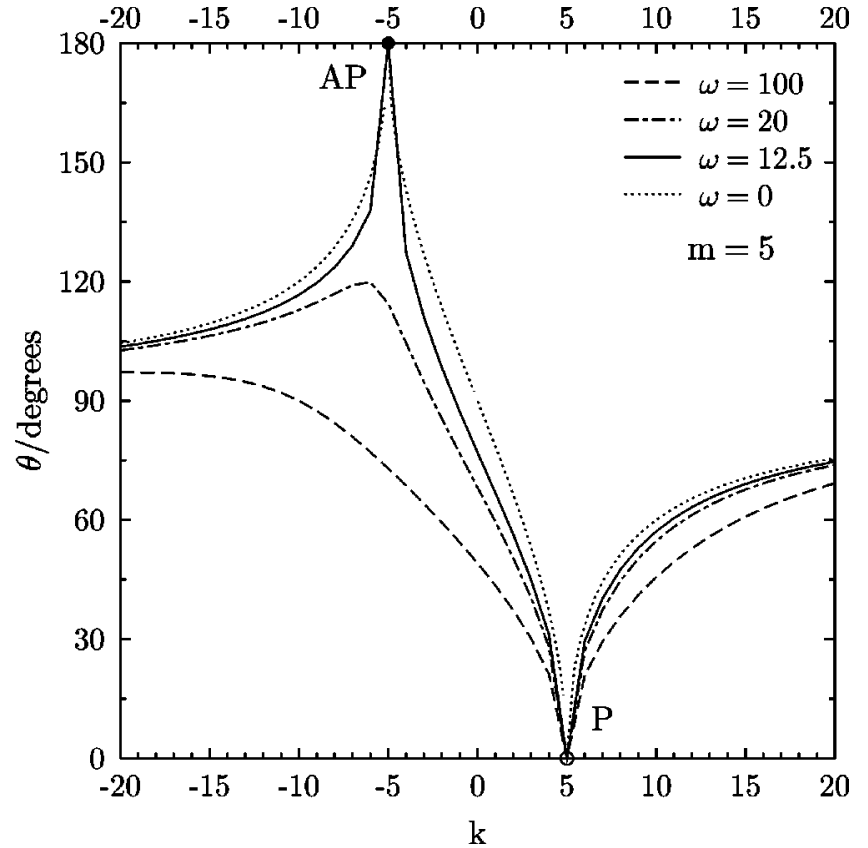

FIG. 1. The angle $\theta$ as a function of $k$ for the tilted $\mathrm{T}^{k}$ relative equilibria for different values of $\omega$ and fixed $m=5$. The parallel P and antiparallel AP relative equilibria are indicated by empty and filled circles, respectively.

librium. When $k=m$ there are no genuine tilted relative equilibria. The same is true for $k=-m$ if $|m| \geqslant \sqrt{2 \omega}$, but if the inequality is reversed there exists a unique tilted relative equilibrium in addition to the anti-parallel relative equilibrium.

This is illustrated in Fig. 1, where the angle $\theta$ is calculated for various $k$ and $\omega$ but fixed projection $m=5$ using Eq. (7). The results for other values of $m$ are qualitatively the same. The parallel and antiparallel relative equilibria are indicated by empty and filled circles respectively. Note that $\theta$ does not depend on the ratio $\gamma=a / b$. When the field is small both $\mathrm{P}$ and AP relative equilibria lie on a curve of tilted relative equilibria. However when $\omega$ exceeds $\mathrm{m}^{2} / 2$, the antiparallel equilibrium bifurcates and becomes isolated. In this latter case the angle $\theta$ for a tilted relative equilibrium achieves a maximum when the ratio $m / k$ is less than -1 . The maximum tends to $\pi / 2$ as the field strength increases.

Among the tilted relative equilibria $\mathrm{T}^{k}$ we distinguish those which give extrema of the energy as $k$ is varied. These correspond to relative equilibria for which the angular velocity about the symmetry axis of the molecule is 0 . Calculations show that for each fixed $m$ and $\omega$ there is at most one of these, which we denote by $\mathrm{T}$. The set of $\mathrm{P}, \mathrm{AP}$, and $\mathrm{T}$ relative equilibria is parameterized by $m$ and can be tuned by the field strength parameter $\omega$.

\section{B. Linear stability of $\mathbf{P}$ and AP relative equilibria}

In this section we discuss the stability of the $\mathrm{P}$ and $\mathrm{AP}$ relative equilibria, considered as equilibrium points of the restriction of the Hamiltonian (2) and equations of motion (3), (4) to $\mathbf{J} . \mathbf{n}=m, \mathbf{n} \cdot \mathbf{n}=1$. Near the P and AP relative equilibria we can take $J_{x}, J_{y}, n_{x}$, and $n_{y}$ as coordinates and the linearized equations of motion are then

$$
\begin{aligned}
\left(\begin{array}{c}
\dot{J}_{x} \\
\dot{n}_{x} \\
\dot{J}_{y} \\
\dot{n}_{y}
\end{array}\right) & =\left(\begin{array}{cccc}
0 & 0 & 2(a-b) m & -b \omega \\
0 & 0 & -\epsilon 2 b & 2 a m \\
-2(a-b) m & b \omega & 0 & 0 \\
\epsilon 2 b & -2 a m & 0 & 0
\end{array}\right) \\
& \times\left(\begin{array}{c}
J_{x} \\
n_{x} \\
J_{y} \\
n_{y}
\end{array}\right)
\end{aligned}
$$

where $\epsilon=+1$ for the parallel relative equilibria and -1 for the antiparallel relative equilibria. A straightforward calculation shows that the normal mode frequencies are the square roots of the eigenvalues of the matrix,

$$
\begin{aligned}
A^{2} & =\left(\begin{array}{cc}
2(a-b) m & -b \omega \\
-\epsilon 2 b & 2 a m
\end{array}\right)^{2} \\
& =4 b^{2}\left(\begin{array}{cc}
(\gamma-1)^{2} m^{2}+\epsilon \omega / 2 & \omega(1-2 \gamma) m / 2 \\
\epsilon(1-2 \gamma) m & \gamma^{2} m^{2}+\epsilon \omega / 2
\end{array}\right) .
\end{aligned}
$$

These are given by

$$
\Omega_{ \pm}^{2}=b^{2}\left[m(2 \gamma-1) \pm \sqrt{m^{2}+2 \epsilon \omega}\right]^{2} .
$$

The relative equilibria are therefore linearly stable if and only if both these expressions are real and positive.

To each of the normal modes with a real frequency we also assign the index $(+1)$ or $(-1)$ according to whether the energy of the normal mode increases or decreases as its amplitude increases. We similarly label unstable normal modes with complex frequencies by (0). Relative equilibria which minimize the energy (for fixed $m$, but variable $k$ ) are necessarily of type $(1,1)$.

For $\gamma \neq 1$ and sufficiently small electric field both $\mathrm{P}$ and AP configurations have only real frequencies and so are linearly stable. If the molecule is oblate then they also minimize the energy and so are both of type $(1,1)$. If the molecule is prolate then they are saddle points of the energy and of type $(1,-1)$. However as the field strength increases the relative equilibria may change stability and undergo bifurcations. The points where these changes occur depend only on $\gamma$ and $\omega / \mathrm{m}^{2}$ and are summarized in Fig. 2.

The frequencies of the $\mathrm{P}$ relative equilibria are always real, $\Omega_{+}$is always strictly positive but $\Omega_{-}$is equal to 0 when $\omega / m^{2}=2 \gamma(\gamma-1)$. The index of the corresponding normal mode is $(+1)$ for $\omega / m^{2}>2 \gamma(\gamma-1)$ and $(-1)$ if the inequality is reversed.

The frequencies of the AP relative equilibria are real if and only if $\omega / m^{2} \leqslant 1 / 2$. If this is satisfied, then $\Omega_{+}$is always strictly positive but $\Omega_{-}$is equal to 0 when $\omega / m^{2}=-2 \gamma(\gamma$ $-1)$. The sign of the corresponding normal mode is $(+1)$ for $\omega / m^{2}<-2 \gamma(\gamma-1)$ and $(-1)$ if the inequality is reversed. At $\omega / \mathrm{m}^{2}=1 / 2$ both normal mode frequencies become equal and for $\omega / \mathrm{m}^{2}>1 / 2$ they are strictly complex and the relative equilibria have type $(0,0)$.

\section{Bifurcations from $P$ and AP relative equilibria}

Each of the stability changes described above has bifurcations associated with it. Along the curve $\omega / m^{2}=2 \gamma(\gamma$ 


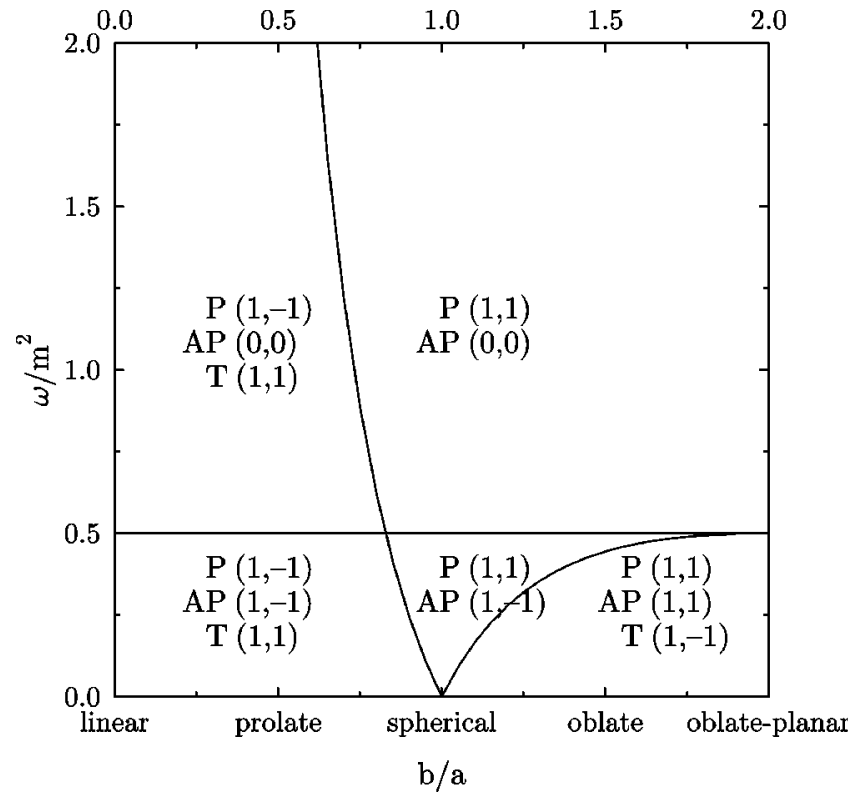

FIG. 2. Bifurcation diagram for symmetric tops in electric fields. Solid lines are defined in Sec. II B. They separate parameter space into five regions. The number of relative equilibria in each region is indicated, together with their stability indices.

-1) a normal mode frequency of the $\mathrm{P}$ relative equilibria goes through 0 and the tilted relative equilibria $\mathrm{T}$ bifurcate. The $\mathrm{T}$ relative equilibria exist for $\omega / m^{2}<2 \gamma(\gamma-1)$ and there they minimize the energy and so have type $(1,1)$. Similarly, along the curve $\omega / m^{2}=-2 \gamma(\gamma-1)$ a normal mode frequency of the AP relative equilibria goes through 0 and again a family of $\mathrm{T}$ relative equilibria bifurcates. These exist for $\omega / m^{2}<-2 \gamma(\gamma-1)$ but are now saddle points of the energy and so have type $(1,-1)$.

A different type of bifurcation occurs when $\omega / \mathrm{m}^{2}$ $=1 / 2$. This is where the AP relative equilibria lose linear stability as a consequence of their normal mode frequencies becoming complex, a phenomenon known as a gyroscopic instability or Hamiltonian Hopf bifurcation. ${ }^{28}$

Figure 3 presents four qualitatively different bifurcation diagrams for the energy dependencies of $\mathrm{P}, \mathrm{AP}$, and T relative equilibria as a function of $m$ with the field strength parameter $\omega$ held constant. Since the bifurcations depend only on the ratio $\omega / m^{2}$, this is equivalent to fixing $m \neq 0$ and varying $\omega$. The diagrams can be regarded as fixed $\gamma$ slices through Fig. 2.

These bifurcations can also be seen in the energymomentum diagrams in Figs. 4 and 5. The solid curves are the energies of the $\mathrm{T}^{k}$ relative equilibria, plotted as functions of $k$ with $m$ held constant in each diagram. The filled circles are the corresponding energy-momentum values of the $\mathrm{P}(k$ $=m)$, $\mathrm{AP}(k=-m)$, and $\mathrm{T}(k \neq \pm m)$ relative equilibria. (The dashes are quantum energy-momentum levels and will be discussed in the next section.)

Figure 4 shows a sequence of energy-momentum diagrams for a prolate molecule $(\gamma=2)$ for fixed $\omega=50$ and increasing $m$. Note that qualitatively this is equivalent to fixing $m$ and decreasing $\omega$. They can be regarded as a succession of two dimensional sections through the analogous three dimensional diagram with energy, $k$ and $m$ along the axes. The full diagram is shown in Refs. 10, 11. As $m$ increases the $\mathrm{P}$ relative equilibrium changes from being a mini-
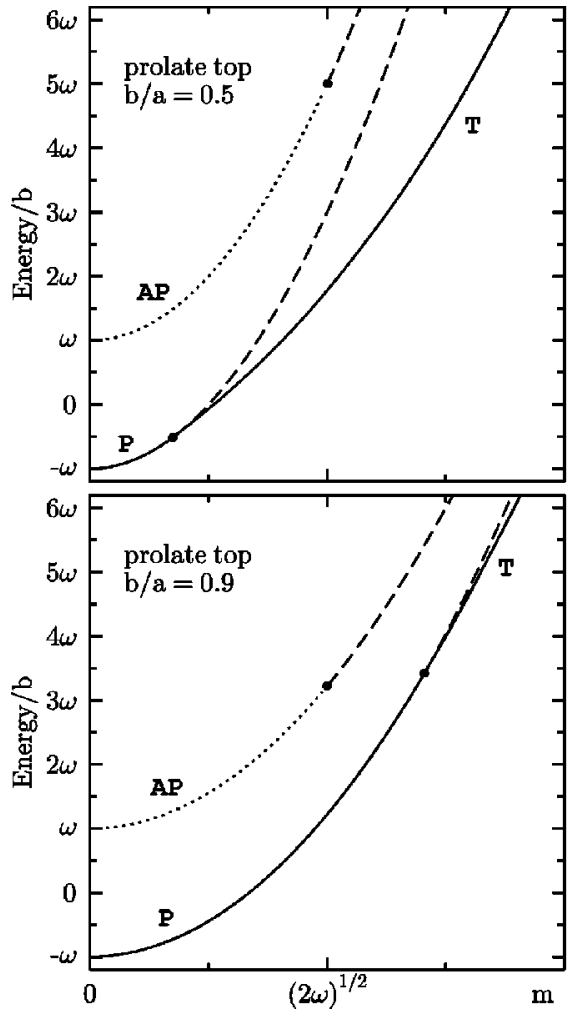

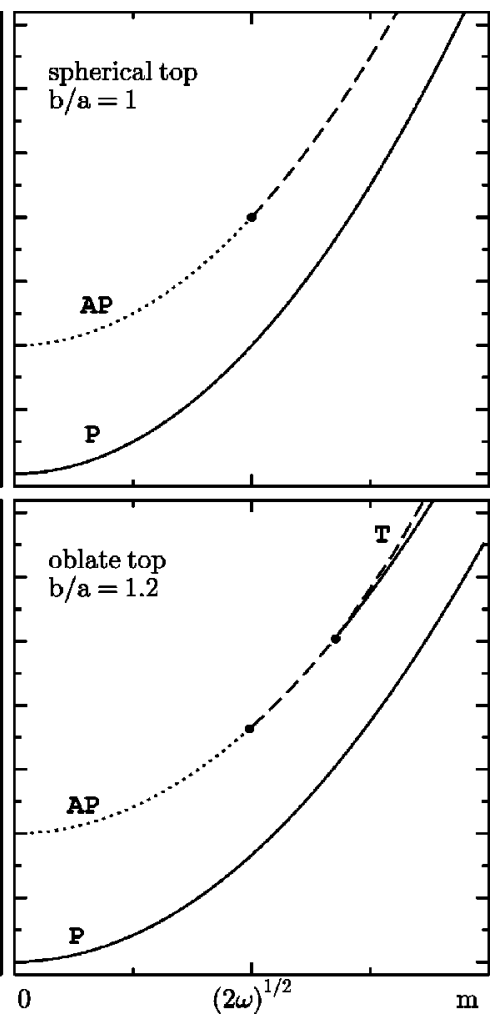

FIG. 3. Energies of P, AP, and T relative equilibria with fixed strength of the electric field and different $m$ : prolate case $b / a=1 / 2$, prolate case $b / a=9 / 10$, spherical case $b / a=1$, oblate case $b / a=6 / 5$. Solid lines denote $(1,1)$ stable relative equilibria, dashed lines $(1,-1)$ linearly stable equilibria, and dotted lines $(0,0)$ unstable equilibria. 

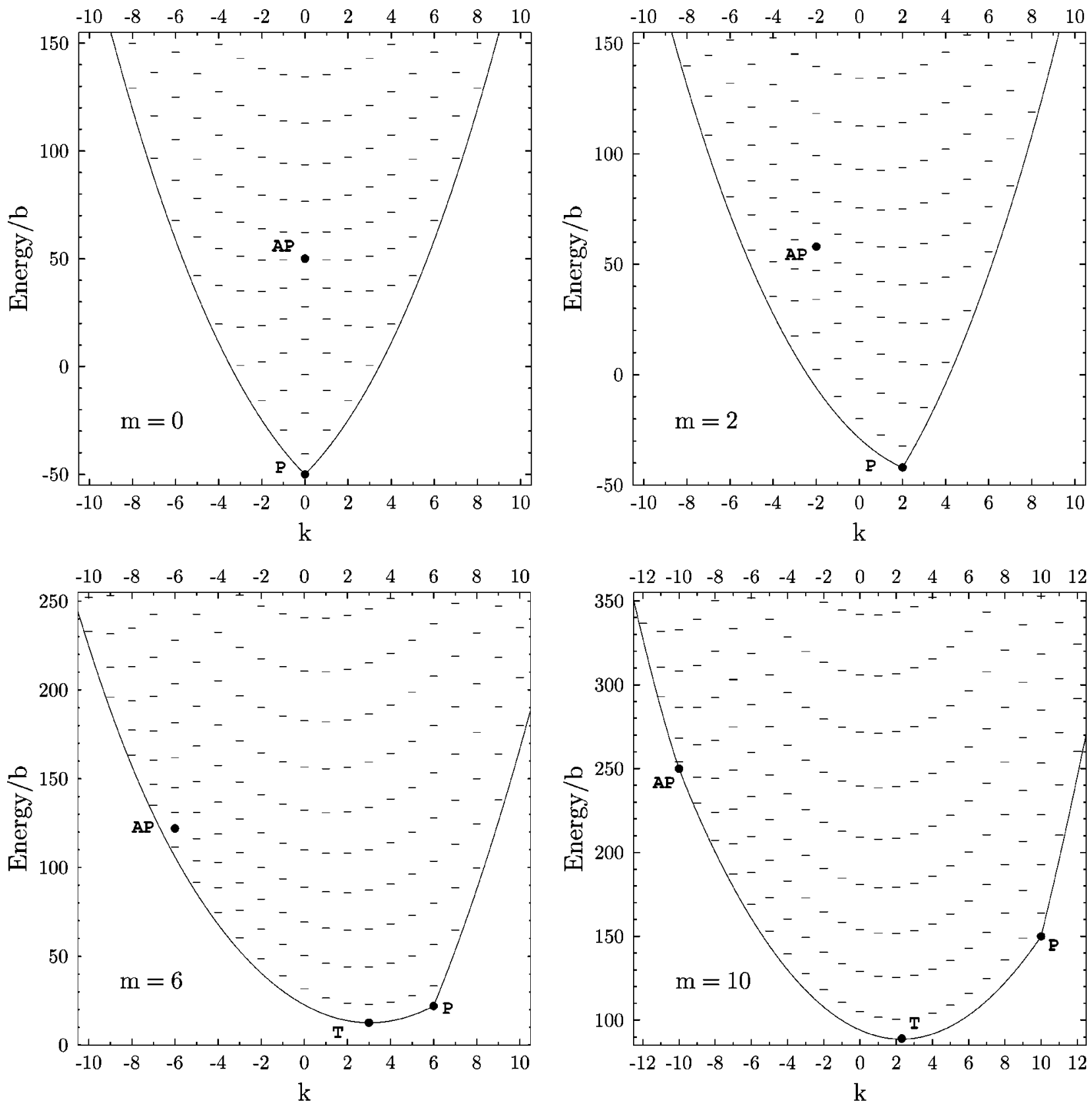

FIG. 4. Quantum energies and classical energy-momentum diagrams of a prolate molecule $(\gamma=a / b=2)$ in the electric field $\omega=50$ for different values of $m$.

mum of the energy to a saddle point as a T relative equilibrium bifurcates from it and becomes a minimum. It follows that a mechanism for selecting specific $m$ states could be a way to create oriented states with different $\theta$ (see Fig. 1) simply by cooling the molecular beam.

Meanwhile the AP relative equilibrium moves closer to the line of $\mathrm{T}^{k}$ relative equilibria and then "sticks" to it. At this point it gains linear stability, though it is still a saddle point of the energy (two real frequencies with opposite signs). This is the Hamiltonian Hopf bifurcation. Figure 5 shows an analogous sequence of diagrams for an oblate molecule $(\gamma=0.8)$ in the same field. In this case the $\mathrm{P}$ relative equilibrium is always a minimum of the energy. The AP relative equilibrium first undergoes a Hamiltonian Hopf bifurcation to stabilize it as a saddle point of the energy, and then becomes a minimum energy point through a bifurcation to a saddle point T relative equilibrium. Similar phenomena have also been observed in a model for the hydrogen atom in crossed electric and magnetic fields ${ }^{22}$ and in a Hamiltonian for two coupled angular momenta. ${ }^{25}$

An approximate expression for the energies $\mathrm{E}\left(\mathrm{T}^{k}\right)$ of the $\mathrm{T}^{k}$ relative equilibria close to the $\mathrm{P}$ relative equilibrium can be obtained by substituting the standard small angle approximation for $\theta$ into Eqs. (7). If $2 \omega+k m \gg(m-k)^{2}$ this yields

$$
\theta^{2} \approx \frac{2|m-k|}{\sqrt{2 \omega+k m}} \ll 1
$$

and hence

$$
\frac{E\left(T^{k}\right)}{b} \approx \sqrt{2 \omega}|m-k|+\gamma k^{2}-\omega .
$$



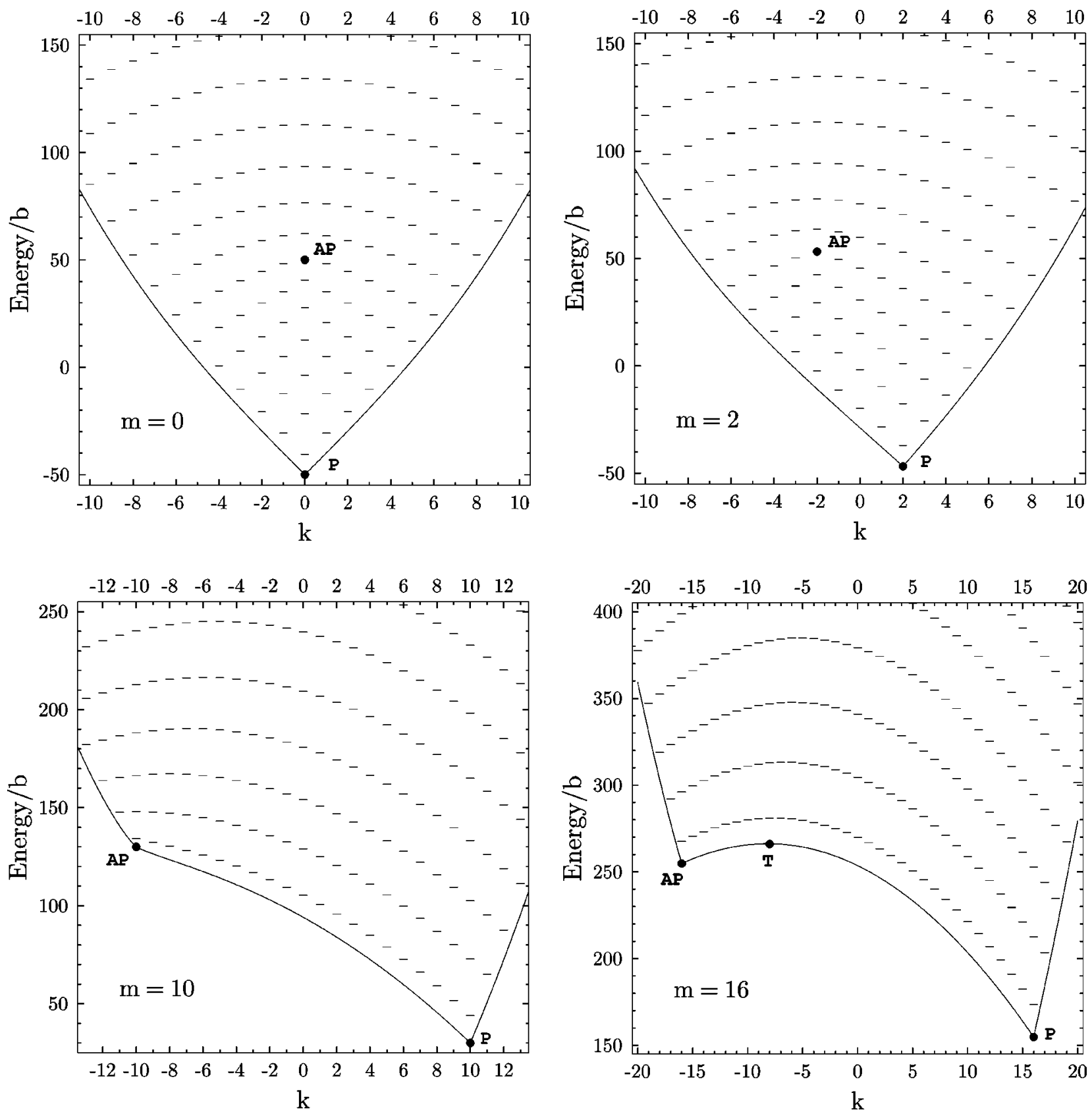

FIG. 5. Quantum energies and classical energy-momentum diagrams of an oblate molecule $(\gamma=a / b=0.8)$ in the electric field $\omega=50$ for different values of $m$.

It is the modulus which creates the "corner" in the energymomentum curve at the P relative equilibrium.

In this discussion we have treated the symmetric top as a two degree of freedom system parametrised by the integral of motion $J_{Z}=m$. The relative equilibria are the critical points of the energy-momentum map $\left(H / b, J_{z}\right)$ from the phase space of this system to the plane. The energymomentum diagrams are plots of the corresponding critical values. The fibers of the energy momentum map at noncritical values are two-dimensional tori. At the critical values they become singular. The singular fibers can be points (equilibria), circles (periodic orbits) or a pinched torus over the AP critical value when this value is isolated. This fiber is a homoclinic connection of the stable and unstable manifolds of the AP equilibrium. The point fibers are the $\mathrm{P}$ relative equilibria and the AP relative equilibria when they form part of the $\mathrm{T}^{k}$ family. The circle fibers are the $\mathrm{T}^{k}$ relative equilibria. The pinched torus is the fiber over the AP critical value when this is isolated from those of the $\mathrm{T}^{k}$ family. The presence of this pinched torus implies that the torus bundle over a circle drawn round the AP critical value is nontrivial. ${ }^{15-18}$ We describe the quantum analog of this classical monodromy in Sec. III. A detailed description of the energy-momentum fibres and classical monodromy of the Lagrange top is given in Ref. 10.

\section{Pendular motion}

With appropriate modifications the discussion above, treating the symmetric top as a two degree of freedom sys- 

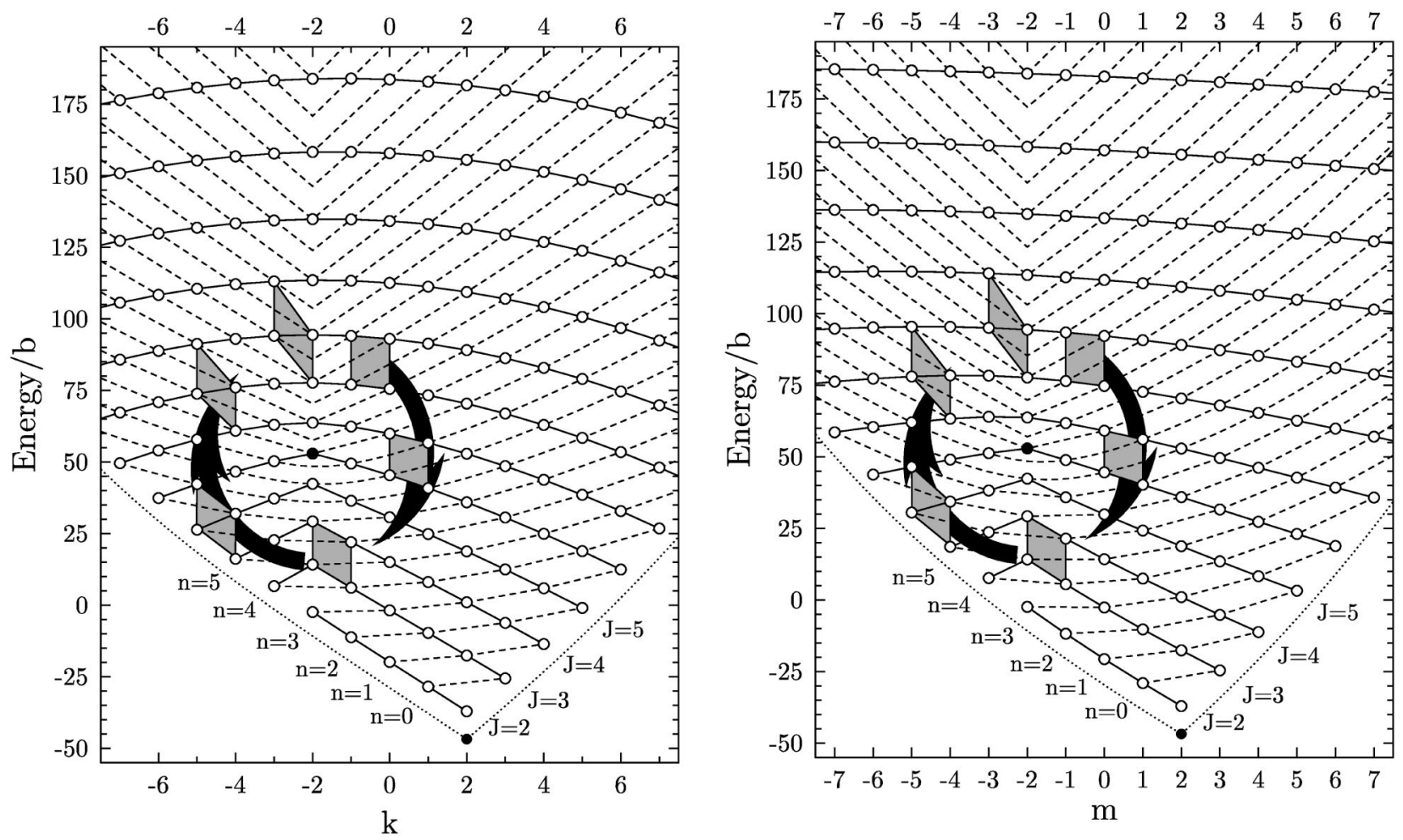

FIG. 6. Quantum energies and classical energy-momentum diagrams of an oblate molecule ( $\gamma=0.8$ ) for fixed $m=2$ (left) and $k=2$ (right) values. The levels with the same $J$ are connected by solid lines while the pendular levels with the same $n$ are connected by dashed lines. Circular translation of the gray quadrilateral around the AP relative equilibrium demonstrates the quantum monodromy effect. See the text for more details.

tem parametrized by the integral of motion $J_{Z}=m$, also works for asymmetric tops. Here we briefly recall the form the Hamiltonian takes when considered as a one degree of freedom system parametrized by the two integrals $J_{Z}=m$ and $J_{z}=k$. Clearly this can only be done in the symmetric top case. The projections of the total angular momentum onto the molecular axes can be expressed in terms of Euler angles $(\theta, \chi, \phi)$ and their conjugate momenta $\left(p_{\theta}, p_{\chi}, p_{\phi}\right){ }^{41}$ In particular,

$$
\begin{aligned}
& J_{Z}=p_{\phi}=m, \\
& J_{z}=p_{\chi}=k, \\
& J^{2}=p_{\theta}^{2}+p_{\chi}^{2}+\frac{\left(p_{\phi}-p_{\chi} \cos \theta\right)^{2}}{\sin ^{2} \theta} .
\end{aligned}
$$

This substitution transforms the Hamiltonian (2) to the onedimensional form,

$$
\frac{H}{b}=p_{\theta}^{2}+\frac{(m-k \cos \theta)^{2}}{\sin ^{2} \theta}-\omega \cos \theta+\gamma k^{2} .
$$

The corresponding dynamics in the effective potential

$$
V_{\mathrm{eff}}=(m-k \cos \theta)^{2} / \sin ^{2} \theta-\omega \cos \theta+\gamma k^{2}
$$

describes the nutational motion of the top. ${ }^{40}$ For a given $m$, $k$, and $\omega$ the minimum of $V_{\text {eff }}$ is given by the $T^{k}$ relative equilibrium.

At high field strengths vibrations about these minima are referred to as pendular oscillations. The frequencies $\Omega_{\text {pen }}$ of low amplitude oscillations can be estimated from the effec- tive potential (14). In particular, for oscillations about $\mathrm{T}^{k}$ when $\theta$ is small we obtain the approximation

$$
\Omega_{\mathrm{pen}} \approx 4 \sqrt{\frac{1}{15}(m-k)^{2}+\frac{1}{4} k m+\frac{1}{2} \omega} .
$$

For $\omega$ sufficiently large this in turn is approximated by

$$
\Omega_{\mathrm{pen}} \approx \sqrt{8 \omega} .
$$

A thorough discussion of this family of one degree of freedom Hamiltonian systems is given in Ref. 10.

\section{QUANTUM SPECTRA}

In addition to showing the energy-momentum values of the classical relative equilibria, Figs. 4, 5, and 6 also show the corresponding values of the quantum states. In this section we discuss some of the key features of these diagrams.

\section{A. Good quantum numbers}

A complete set of exact quantum numbers is obtained by supplementing $k$ and $m$ by the quantum number $\nu$ for the one-dimensional reduced Hamiltonian (13). This counts the nodes of the corresponding wave functions in the $\theta$ direction.

Quantum states with $E / b \gg \omega$ are given approximately by the free rigid body Hamiltonian $H / b=\mathbf{J}^{2}+(\gamma-1) J_{z}^{2}$. For these states the total angular momentum $J$ is approximately conserved and so they can also be labeled by $(J, k, m)$. In this limit, the relationship between $(J, k, m)$ and $(k, m, \nu)$ is given by

$$
J=(|m+k|+|m-k|) / 2+\nu .
$$


The energies of the quantum states in this limit are given approximately by

$$
E(J, k, m) / b=J^{2}+(\gamma-1) k^{2} .
$$

In this approximation each level is $(2 J+1)$ times degenerate, the states within a given level set being labeled by $m$ $=-J, \ldots, J$. The relationship (17) is clearly visible in Figs. 4 and 5. For prolate molecules (Fig. 4) the high energy states with constant $J$ form parabolas which curve upwards $(\gamma$ $>1$ ), while for oblate molecules (Fig. 5) they form parabolas which curve downwards $(\gamma<1)$. The validity of the free rotor approximation is particularly apparent in Fig. 6, where the energy levels for $\gamma=0.8$ and $\omega=50$ are plotted against both $k$ for fixed $m=2$ and against $m$ for fixed $k=2$. It is clearly seen that the top levels form almost straight lines. This signifies degeneracy in $m$ because the levels project nearly to one point on the energy axis.

For the lowest energy states with given quantum numbers $k$ and $m$ we can apply a quantum harmonic approximation to the one-dimensional system (13) to obtain the following estimate for their energies:

$$
\frac{E(k, m, \nu)}{b} \approx \frac{E\left(T^{k}\right)}{b}+\left(\nu+\frac{1}{2}\right) \Omega_{\mathrm{pen}} .
$$

This in turn can be used to describe the quantum states that are localized near the $\mathrm{P}$ relative equilibrium. These are states with both $|m-k|$ and $\nu$ sufficiently small. When $|m-k|$ is small the angle $\theta$ is close to zero (see Fig. 1) and so the small angle approximations for $\mathrm{E}\left(\mathrm{T}^{k}\right)$ and $\Omega_{\text {pen }}$ provided by Eqs. (11) and (15) can be used to obtain

$$
\frac{E(k, m, \nu)}{b} \approx \sqrt{2 \omega}(2 \nu+1+|m-k|)+\gamma k^{2}-\omega .
$$

This is consistent with the classical analysis of the parallel relative equilibrium since its normal mode frequencies, given by Eq. (9), degenerate into those of a 2D isotropic oscillator in the strong field limit. Indeed, by analogy one can replace $\nu$ by a pendular quantum number

$$
n=2 \nu+|m-k|
$$

and so obtain the spectrum

$$
\frac{E(k, n)}{b} \approx \sqrt{2 \omega}(n+1)+\gamma k^{2}-\omega .
$$

Each energy level is approximately $n+1$ times degenerate: for fixed values of $n, k$ the states with $m, \nu$ satisfying $\nu$ $=0, \ldots,[n / 2], m=k \pm(n-2 \nu)$ all have the same approximate energy. Here $[n / 2]$ denotes the integer part of $n / 2$. Note that states with the same $n$ will generally have different nodal structures because they may have different values of $\nu$. The upward curving parabolas formed by states with the same value of $n$ are clearly visible in Figs. 4 and 5 near the $P$ relative equilibrium.

Although the approximate spectra given by Eqs. (17) and (20) are only valid for free and localized states, respectively, the labelling by quantum numbers $(J, k, m)$ and $(k, m, n)$ can be extended to all states by using Eqs. (16) and (19). Eliminating $\nu$ from these equations gives the relationship between $J$ and $n$,

$$
n=2 J-|m+k| \text {. }
$$

One may think of Eq. (21) as an extension, with the additional parameter $k$, to the similar relationship in the spherical pendulum model where it has the form $n=2 J-|m|$ (see Ref. 12, Fig. 4). The resulting constant $J$ and constant $n$ families of states are illustrated in Fig. 6 by solid and dashed lines, respectively. The constant $J$ lines are smooth above the AP relative equilibrium, but have a kink below it. In other words, along the curves below the AP relative equilibrium the derivative of the energy with respect to $k$ (or $m$ ) has a discontinuity at $k=-m$. A similar discontinuity occurs along the constant pendular number $n$ curves above the AP relative equilibria.

\section{B. Monodromy}

The quantum effect of monodromy is illustrated in Fig. 6 , which shows the energy levels of a symmetric top with $\gamma=0.8$ in a field $\omega=50$. In the first panel the energy levels are plotted against $k$ while the angular momentum projection onto the field direction is fixed at $m=2$. In the other panel the angular momentum projection onto the symmetry axis is fixed at $k=2$ while $m$ is allowed to vary. Both diagrams illustrate the effect of translating a unit cell of the lattice of energy-momentum points about the AP relative equilibrium: the cell does not return to itself after a complete circuit. If an analogous operation is performed around a circuit that does not include the AP point then the cell would return to itself. It follows from this that there is no possible choice of quantum numbers which can work globally in the whole neighborhood around AP point. This phenomenon was first described for the quantum spherical pendulum by Cushman and Duistermaat, ${ }^{21}$ for the champagne bottle potential by Child $^{13}$ and for the hydrogen atom in crossed fields by Cushman and Sadovskii. ${ }^{22}$ A rigorous general account has been given by $\mathrm{Vu} \mathrm{Ngoc} .^{23,24}$ A semiclassical analysis of the relationship between monodromy and the kinks in the constant $J$ and constant $n$ curves of quantum states is given in Refs. 12 and 13, Zhilinskii and Sadovskii have established a connection between monodromy and energy level redistribution ${ }^{25}$ and described monodromy as a defect in the lattice of energy levels in the energy-momentum diagram.

When $k=0$ the Lagrange top degenerates to a system that is equivalent to the spherical pendulum. The plot of energy against $m$ in this case is therefore the same as the diagram in Ref. 21 and Fig. 4 of Ref. 12, and is very similar to the $m=0$ diagram with energy plotted against $k$ for oblate molecules shown in the first panel of Fig. 5 of this paper. All the $k=0$ and $m=0$ diagrams share the property that they have a reflectional symmetry and the AP relative equilibrium is a monodromy point lying on the axis of symmetry above the $\mathrm{P}$ relative equilibrium. However, as $k$ or $m$ is moved away from zero this reflectional symmetry is broken and the AP relative equilibrium moves towards the boundary curve consisting of $\mathrm{T}^{k}$ relative equilibria. As described in Sec. II C the Hamiltonian Hopf bifurcation corresponds to the point where the AP relative equilibrium reaches the boundary curve and "sticks" to it. The AP relative equilibrium remains a classical monodromy point for as long as it remains iso- 
lated from the $\mathrm{T}^{k}$ relative equilibria, and quantum monodromy can be observed provided there are enough quantum states surrounding the AP point to construct a family of unit cells. However after the Hamiltonian Hopf bifurcation both classical and quantum monodromy disappear and uniform labelling of quantum states by either of the sets of quantum numbers $(J, k, m)$ or $(k, m, n)$ is possible.

\section{Quasisymmetric top}

To observe the phenomena described above in a real quasisymmetric molecule it is necessary that they persist when the symmetry of the molecule is broken slightly. The main problem here is the construction of the energy level lattice or, equivalently, the definition of appropriate (approximate) quantum numbers. One approach is to perturb away from the symmetric top molecule with the field turned on and label the quasi-symmetric states by the quantum numbers of the symmetric states they correlate back to. An example of this is given in Fig. 7 which shows the energy levels of a prolate symmetric top (as given in Fig. 4) together with the levels of an asymmetric top with similar rotational constants. Although there is some distortion to the lattice it is clear that the main qualitative features persist. In particular the region of pendular states seems to be almost unaffected by the perturbation and the pendular quantum number is still useful in the region of localization.

A second approach to constructing an energy level lattice for asymmetric molecules in an electric field is to perturb away from the zero field limit, while preserving the rotational constants, and label the perturbed states by the quantum numbers of the free rigid body states they correlate to. This is the approach taken in Ref. 9 and applied successfully to the quantum states of acetylene-HF in strong electric fields. Their pendular group number $n=2 J-\mid M$ $+(-1)^{\sigma} K_{a} \mid$, where $\sigma=J+1-K_{a}-K_{c}$, is completely analogous to our pendular number $n$ given by Eq. (21). For the example of Fig. 7 this perturbation shows that the structure of the upper energy levels in the diagram is very close to the free rotation structure for a molecule with the same rotational constants.

Analogs of much of the classical skeleton described in Sec. II persist for general asymmetric rigid molecules in electric fields. In particular the P, AP, and T relative equilibria remain, though their bifurcations are different. ${ }^{36-38}$ Moreover the $\mathrm{T}^{k}$ relative equilibria, which form the boundaries of the energy-momentum diagrams, persist as relative periodic orbits. In future work we hope to analyze the way these classical trajectories organize the quantum states of general asymmetric molecules.

\section{Critical field strength}

Figures 4, 5, and 6 given above present fixed $m$ or fixed $k$ slices through the total three-dimensional system of levels which lies over the $(m, k)$ plane. If presented in this way, the AP relative equilibria form a line which passes through the three-dimensional energy level structure. ${ }^{10,11,26}$ In this sense classical monodromy exists at any field strength $\mathcal{E} \neq 0$. However quantum monodromy will only be observable if the

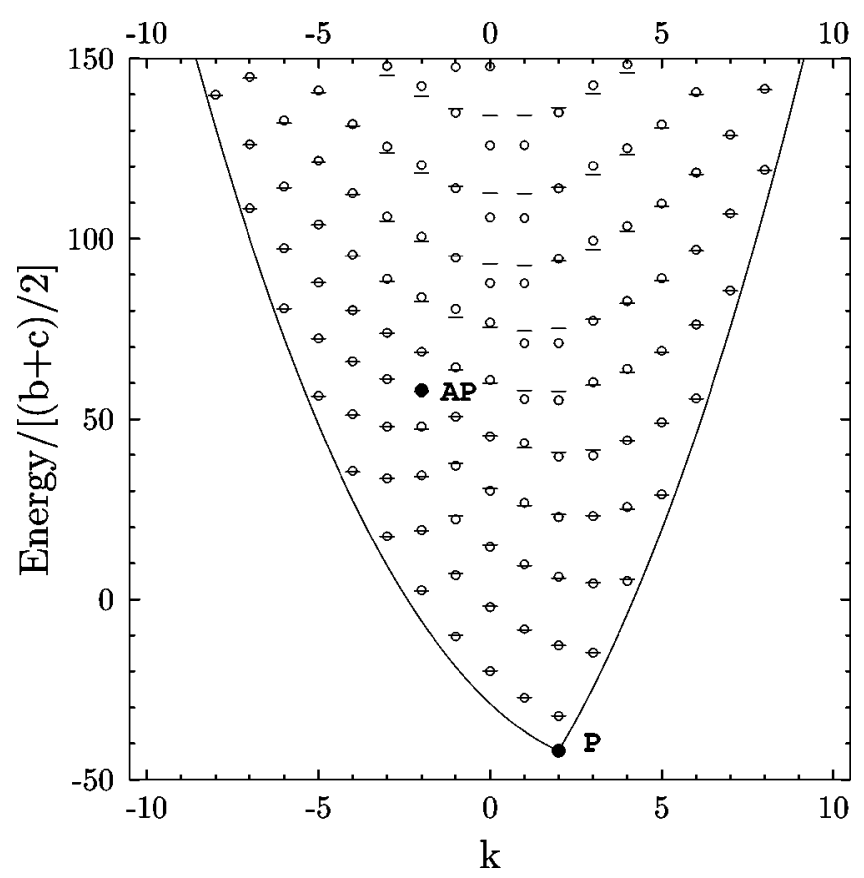

FIG. 7. Energy levels of an asymmetric top molecule with $a=2, b=1.1$, $c=0.9$ are shown as circles for $m=2$ and field strength $\omega=50$. For comparison the energy levels of a symmetric top molecule with $a=2, b=c$ $=1$ in the same field are also presented, together with its classical energymomentum map.

monodromy point is surrounded by a sufficient number of levels. In particular at least two levels should be located between $\mathrm{P}$ and AP relative equilibria when $k=m=0$. This means that the energy of the first excited state, which is approximately $3 \sqrt{2 \omega}$ above the parallel configuration, must be less than that of the AP relative equilibrium. Since the energy difference between $\mathrm{P}$ and AP relative equilibria is equal to $2 \omega$, we obtain a field strength estimate of $\omega>4.5$ for quantum monodromy to be observable.

The experimental value of $\omega$ achieved for $\mathrm{C}_{2} \mathrm{H}_{2}-\mathrm{HF}$ in Ref. 9 is 4.82 , which is slightly above the critical value. However much greater values of $\omega$ have been achieved in a number of other recent experiments. For example, $\omega=24.2$ was obtained for the near prolate molecule $\mathrm{C}_{6} \mathrm{H}_{5} \mathrm{I}$ in a 20 $\mathrm{kV} / \mathrm{cm}$ electric field, ${ }^{7}$ and $\omega=38$ was achieved in a 57 $\mathrm{kV} / \mathrm{cm}$ field for the near oblate molecule pyridazine. ${ }^{8}$ Fields of up to $100 \mathrm{kV} / \mathrm{cm}$ have been reported in orientation experiments. ${ }^{5}$ However large values of $\omega$ can also be achieved when rotational constants are small. A record value of $\omega=365$ has been obtained for the linear trimer $(\mathrm{HCN})_{3}$ with a field strength of about $30 \mathrm{kV} / \mathrm{cm}$ because of its extremely big dipole moment and very small rotational constant. ${ }^{4}$ Although the monodromy effect has been demonstrated to exist in the rotation-vibration energy level structure of water ${ }^{12}$ the monodromy point is located too high in energy to be experimentally accessible. The Stark field experiments give an occasion where quantum monodromy can be in reach of the modern experimental technique.

\section{E. Localized states}

We have shown elsewhere ${ }^{33,34}$ that relative equilibria can be used to obtain new approximations for the qualitative 
analysis of quantum levels. This can be done for any relative equilibrium but the most experimentally relevant for the Stark effect is the P relative equilibrium. Unlike our previous use of quantum harmonic approximation around classical relative equilibrium (see above and Refs. 33 and 34), here an approximate model will be derived directly from a quantum Hamiltonian.

The quantum Hamiltonian corresponding to Eq. (2) is obtained if $J^{2}$ and $J_{z}^{2}$ are replaced by their respective operators. Assuming the solution has the form $\Psi$ $=\Phi(\theta) \exp (i m \phi) \exp (i k \chi)$, one obtains

$$
\begin{aligned}
& {\left[\frac{\partial^{2}}{\partial \theta^{2}}+\frac{\cos \theta}{\sin \theta} \frac{\partial}{\partial \theta}-\frac{(m-k \cos \theta)^{2}}{\sin ^{2} \theta}-\gamma k^{2}+\omega \cos \theta\right.} \\
& \left.+\frac{E}{b}\right] \Phi=0 .
\end{aligned}
$$

Note that in fact the simple harmonic approximation used above [see Eq. (13)] is almost identical apart from the second term in Eq. (22). Its effect is equivalent to adding a term to the effective potential $V_{\text {eff }}(\theta)$.

Since we are interested in the states localized around $\mathrm{P}$ relative equilibrium we assume $\theta \ll 1$ and expand $\cos \theta$ and $\sin \theta$ around $\theta=0$. Defining a new energy $\widetilde{E}=\omega-\gamma k^{2}$ $-\left(m k-2 k^{2}+m^{2}\right) / 3+E / b$ and a new coordinate $r$ $=\theta \sqrt[4]{\omega / 2}$, simplified Eq. (22) takes the form

$$
\left[\frac{\partial^{2}}{\partial r^{2}}+\frac{1}{r} \frac{\partial}{\partial r}-\frac{l^{2}}{r^{2}}-r^{2}+\widetilde{E} \sqrt{\frac{2}{\omega}}\right] \Phi=0,
$$

where $l=|m-k|$. This is easily recognized as the standard radial form of an isotropic 2D oscillator. Also note that Eq. (23) is relevant for rotating quasilinear molecules, ${ }^{43}$ which exhibit monodromy. ${ }^{12}$ For the energy,

$$
\begin{aligned}
\frac{E}{b}= & \sqrt{2 \omega}(2 n+|m-k|+1)-\omega+\gamma k^{2} \\
& +\frac{1}{3}\left(m k-2 k^{2}+m^{2}\right)
\end{aligned}
$$

the corresponding wave function is

$$
\Phi_{n, l}(r)=N_{n, l} e^{-r^{2} / 2} r^{l} L_{n+l}^{l}\left(r^{2}\right),
$$

where $N_{n, l}$ is a normalization factor and $L_{n+l}^{l}\left(r^{2}\right)$ is the associated Laguerre polynomial of degree $r^{2 n}$. Because $\theta$ exists in the region from 0 to $\pi$ the orthogonality condition holds only approximately

$$
\int_{0}^{\pi} \sqrt{\frac{\omega}{2}} \Phi_{n, l}\left(\theta \sqrt[4]{\frac{\omega}{2}}\right) \Phi_{n^{\prime}, l}\left(\theta \sqrt[4]{\frac{\omega}{2}}\right) \theta d \theta \approx \delta_{n^{\prime}, n} .
$$

The probability distribution along $\theta$ is $\sqrt{(\omega / 2)} \Phi_{n, l}^{2}(\theta \sqrt[4]{\omega / 2}) \theta$ with $n$ peaks. Note that the $n$ is essentially the same as that used in the harmonic approximation. The distribution becomes higher and narrower, shrinking to $\theta=0$, as the field increases, thus validating our strong field approximation. The total wave function is then

$$
|n k m\rangle=\sqrt[4]{\frac{\omega}{2}} e^{i m \phi} e^{i k \chi} \Phi_{n, l}\left(\theta \sqrt[4]{\frac{\omega}{2}}\right) .
$$

The line strength of a transition due to a permanent dipole moment (or transition moment) along the symmetry axis is equal to ${ }^{41}$

$$
\begin{aligned}
S_{i j}= & |\langle i|\sin \theta \cos \phi| j\rangle|^{2}+|\langle i|\sin \theta \sin \phi| j\rangle|^{2} \\
& +|\langle i|\cos \theta| j\rangle|^{2} .
\end{aligned}
$$

The first two terms in the sum come from the $\Delta m= \pm 1$, $\Delta k=0$ transitions, which are proportional to the square of

$$
\begin{aligned}
& \sqrt{\frac{\omega}{2}} \int_{0}^{\pi} \Phi_{n, l}\left(\theta \sqrt[4]{\frac{\omega}{2}}\right) \Phi_{n^{\prime}, l+1}\left(\theta \sqrt[4]{\frac{\omega}{2}}\right) \sin ^{2} \theta d \theta \\
& \approx \sqrt[4]{\frac{2}{\omega}} \int_{0}^{\infty} \Phi_{n, l}(r) \Phi_{n^{\prime}, l+1}(r) r^{2} d r .
\end{aligned}
$$

The integral is nonzero and equal to $n+l+1$ only when $\Delta n=0$. However the line strength decreases as $1 / \sqrt{\omega}$ as the field grows. Other possible transitions, which arise due to the third term in Eq. (26), have selection rule $\Delta m=0, \Delta k=0$. These are proportional to the square of

$$
\begin{aligned}
& \sqrt{\frac{\omega}{2}} \int_{0}^{\pi} \Phi_{n, l}\left(\theta \sqrt[4]{\frac{\omega}{2}}\right) \Phi_{n^{\prime}, l+1}\left(\theta \sqrt[4]{\frac{\omega}{2}}\right) \sin \theta \cos \theta d \theta \\
& \approx \int_{0}^{\infty} \Phi_{n, l}(r) \Phi_{n^{\prime}, l}(r) r d r .
\end{aligned}
$$

Because of the orthogonality condition this integral is nonzero only when $\Delta n=0$, implying no changes at all to the quantum numbers. Thus a strong electric field completely destroys the pure rotational transitions between localized states. This should not come as a surprise, because it is physically clear that in a rotational transition a molecule must change the way it rotates, and a molecule in an oriented state is strongly prevented from doing so by the field. However the field does not suppress rovibrational transitions between the same $n, m, k$.

Moore, Oudejans, and Miller ${ }^{9}$ have given excellent examples of the changes induced by a strong electric field in the rovibrational spectrum. In Figs. 3 and 4 of that paper the calculated spectrum of the HF stretch in $\mathrm{C}_{2} \mathrm{H}_{2}-\mathrm{HF}$ is presented for different electric fields. When the field reaches $160 \mathrm{kV} / \mathrm{cm}$ all populated states in their simulation are localized and the spectrum has only distinct pendular features which are assigned pendular quantum numbers. The complex $\mathrm{C}_{2} \mathrm{H}_{2}-\mathrm{HF}$ can be considered a quasisymmetric molecule since $\quad \bar{B}=(B+C) / 2=0.15 \mathrm{~cm}^{-1} \gg(B-C) / 2=0.01 \mathrm{~cm}^{-1}$ and we may apply the above approximation to describe localized states. The frequency yields

$$
\begin{aligned}
\nu \approx & \nu_{1}-\mathcal{E}\left(\mu_{1}-\mu_{0}\right)+\sqrt{2 \mathcal{E}}\left(\sqrt{\bar{B}_{1} \mu_{1}}-\sqrt{\bar{B}_{0} \mu_{0}}\right) \\
& \times(n+1)+\left(A_{1}-A_{0}-B_{1}+B_{0}\right) k^{2},
\end{aligned}
$$

where the subscripts 0 and 1 are used to distinguish the ground state and an excited state respectively. In the first approximation the spectrum degenerates to a single line at the vibrational frequency $\nu_{1}$. But in reality the dipole mo- 
ments and rotational constants in vibrational states are slightly different. Since $\mu_{1}>\mu_{0}$ the Stark field gives a uniform linear red shift of the whole pendular band as the field increases. ${ }^{9}$ In the next approximation there will be a series of equidistant pendular lines with different $n=0,1,2, \ldots$. One can easily calculate the spacing between the lines at $\mathcal{E}$ $=160 \mathrm{kV} / \mathrm{cm}$ using the $\mathrm{C}_{2} \mathrm{H}_{2}-\mathrm{HF}$ constants given in Ref. 42 and the third term on the right-hand side of Eq. (27). This gives $0.072 \mathrm{~cm}^{-1}$, which compares very favorably with the distance of approximately $0.07 \mathrm{~cm}^{-1}$ between pendular peaks seen in Fig. 4 of Ref. 9. Note that the intensity of the simulated spectrum decreases with $n$ due to thermal population.

\section{DISCUSSION}

This paper has shown how relative equilibria organize the spectrum of a molecule in an electric field and that monodromy arises naturally in the problem of labelling the quantum states. The case of symmetric top molecules is particularly easy to analyze because it is integrable and so labelling is possible by exact quantum numbers. However the effect of monodromy seems quite robust and is likely to be observable even in moderately asymmetric molecules using quantum numbers obtained by correlation back to the symmetric case.

We have also shown that for symmetric tops Hamiltonian Hopf bifurcations provide a natural boundary to the parameter region in which monodromy exists. This phenomenon will also persist for quasisymmetric molecules. Moreover it seems likely that Hamiltonian Hopf bifurcations can be used to demonstrate the existence of monodromy in quantum states that are localized near relative equilibria even in systems that are globally far from integrable. To see this recall that linearly stable relative equilibria organize regular series of localized quantum states that can be labeled by quantum numbers derived from local integrable harmonic approximations to the Hamiltonian. ${ }^{33,34}$ Examples of this include pendular states of rigid molecules in electric fields which are far from the symmetric top or zero field integrable limits. This harmonic approximation can be extended beyond a Hamiltonian Hopf point at which the relative equilibrium loses stability by using a normal form approximation ${ }^{28}$ which is again integrable and so introduces approximate conserved quantities and quantum numbers. For example, the normal form analysis of the rotating equilateral triangle relative equilibrium of $\mathrm{H}_{3}^{+}$(Ref. 33) near its Hamiltonian Hopf point shows that the vibrational angular momentum of the molecule is approximately conserved for the dynamics near the relative equilibrium, though of course it is not globally conserved. Using these approximate quantum numbers it should be possible to construct energy-momentum lattices which will have monodromy after the relative equilibrium has lost stability. Qualitatively the "birth" of monodromy in these cases will be the same as that demonstrated in Figs. 4 and 5 near the point where the AP relative equilibrium detaches itself from the curve of $\mathrm{T}^{k}$ relative equilibria. In nonintegrable systems the $\mathrm{T}^{k}$ relative equilibria are themselves replaced by a family of (relative) periodic orbits that bifurcates from the relative equilibrium. ${ }^{28}$

\section{ACKNOWLEDGMENTS}

We are grateful to Richard Cushman, Bretislav Friedrich, Dmitrii Sadovskii, and Boris Zhilinskii for several very useful comments on earlier versions of this paper. This work was supported by a UK EPSRC research grant.

${ }^{1}$ H. J. Loesch and A. Remscheid, J. Chem. Phys. 93, 4779 (1990).

${ }^{2}$ B. Friedrich and D. R. Herschbach, Z. Phys. D: At., Mol. Clusters 18, 153 (1991).

${ }^{3}$ J. M. Rost, J. C. Griffin, B. Friedrich, and D. R. Herschbach, Phys. Rev. Lett. 68, 1299 (1992).

${ }^{4}$ P. A. Block, E. J. Bohac, and R. E. Miller, Phys. Rev. Lett. 68, 1303 (1992).

${ }^{5}$ B. Friedrich, H.-G. Rubahn, and N. Sathyamurthy, Phys. Rev. Lett. 69, 2487 (1992).

${ }^{6}$ J. Bulthuis, J. Möller, and H. J. Loesch, J. Phys. Chem. A 101, 7684 (1997).

${ }^{7}$ H. J. Loesch and J. Möller, J. Phys. Chem. A 101, 7534 (1997).

${ }^{8}$ H. Li, K. J. Franks, R. J. Hanson, and W. Kong, J. Phys. Chem. A 102, 8084 (1998)

${ }^{9}$ D. T. Moore, L. Oudejans, and R. E. Miller, J. Chem. Phys. 110, 197 (1999).

${ }^{10}$ R. Cushman and L. Bates, Global Aspects of Classical Integrable Systems (Birkhäuser, Basel, 1997).

${ }^{11}$ R. Cushman and H. Knörrer, in Differential Geometric Methods in Mathematical Physics (Proceedings, Clausthal, 1983), Lecture Notes in Mathematics, edited by H. D. Doebner and D. J. Hennig (Springer-Verlag, Berlin, 1985), Vol. 1139, p. 12.

${ }^{12}$ M. S. Child, T. Weston, and J. Tennyson, Mol. Phys. 96, 371 (1999).

${ }^{13}$ M. S. Child, J. Phys. A 31, 657 (1998).

${ }^{14}$ J. J. Duistermaat, Commun. Pure Appl. Math. 33, 687 (1980).

${ }^{15}$ M. Zou, J. Geom. Phys. 10, 37 (1992).

${ }^{16}$ V. S. Matveev, Sbornik Math. 187, 495 (1996).

${ }^{17}$ N. Tien Zung, Diff. Geom. Applic. 7, 123 (1997).

${ }^{18}$ R. Cushman and J. J. Duistermaat, J. Diff. Eqns. 172, 42 (2001).

${ }^{19}$ L. Bates, ZAMP 42, 837 (1991).

${ }^{20}$ R. H. Cushman, Centrum voor Wiskunde en Informatica Newsletter, 1, 4 (1983).

${ }^{21}$ R. Cushman and J. J. Duistermaat, Bull. Am. Math. Soc. 19, 475 (1988).

${ }^{22}$ R. Cushman and D. A. Sadovskii, Europhys. Lett. 47, 1 (1999); Physica D 142, 166 (2000)

${ }^{23}$ S. Vu Ngoc, Commun. Math. Phys. 203, 465 (1999).

${ }^{24}$ S. Vu Ngoc, Commun. Pure Appl. Math. 53, 143 (2000).

${ }^{25}$ D. A. Sadovskii and B. I. Zhilinskii, Phys. Lett. A 256, 235 (1999).

${ }^{26}$ L. Grondin, D. A. Sadovskii, and B. I. Zhilinskii, Phys. Rev. A 65, 012105 (2001).

${ }^{27}$ M. P. Jacobson and M. S. Child, J. Chem. Phys. 114, 262 (2001).

${ }^{28}$ J. C. van der Meer, Hamiltonian Hopf Bifurcation, Lecture Notes in Mathematics (Springer-Verlag, Berlin, 1985), Vol. 1160.

${ }^{29}$ J. J. Duistermaat, ZAMP 48, 1 (1997).

${ }^{30}$ W. G. Harter, Comput. Phys. Rep. 8, 319 (1988).

${ }^{31}$ I. M. Pavlichenkov, Phys. Rep. 226, 175 (1993).

${ }^{32}$ B. I. Zhilinskii, Phys. Rep. 341, 85 (2001).

${ }^{33}$ I. N. Kozin, R. M. Roberts, and J. Tennyson, J. Chem. Phys. 111, 140 (1999).

${ }^{34}$ I. N. Kozin, R. M. Roberts, and J. Tennyson, Mol. Phys. 98, 295 (2000).

${ }^{35} \mathrm{R}$. Cushman and J. C. van der Meer, in Géométrie Symplectique et Mécanique (Proceedings, La Grande Motte, 1988), Lecture Notes in Mathematics, edited by C. Albert (Springer-Verlag, Berlin, 1990), Vol. 1416, p. 26.

${ }^{36}$ S. B. Katok, Usp. Mat. Nauk 27, 126 (1972).

${ }^{37}$ Ya. V. Tatarinov, Vestnik Moskov. Univ. 29, 99 (1974).

${ }^{38}$ D. Lewis, T. Ratiu, J. C. Simo, and J. E. Marsden, Nonlinearity 5, 1 (1992).

${ }^{39}$ V. I. Arnold, V. V. Kozlov, and A. I. Neishtadt, Mathematical Aspects of Classical and Celestial Mechanics (Springer-Verlag, Berlin, Heidelberg, 1997).

${ }^{40}$ L. D. Landau and E. M. Lifshitz, Mechanics (Pergamon, Oxford, 1965).

${ }^{41}$ E. B. Wilson, Jr., J. C. Decius, and P. C. Cross, Molecular Vibrations (McGraw-Hill, New York, 1955).

${ }^{42}$ Z. S. Huang and R. E. Miller, J. Chem. Phys. 86, 6059 (1987).

${ }^{43}$ W. Quapp and B. Winnewisser, J. Math. Chem. 14, 259 (1993). 